\title{
Assembly of Spinach Chloroplast ATP Synthase Rotor Ring Protein-Lipid Complex
}

\author{
Olga Novitskaia ${ }^{1}$, Pavel Buslaev ${ }^{1,2}$ and Ivan Gushchin ${ }^{1 *}$ \\ ${ }^{1}$ Research Center for Molecular Mechanisms of Aging and Age-Related Diseases, Moscow Institute of Physics and \\ Technology, Dolgoprudny, Russia, ${ }^{2}$ Nanoscience Center, Department of Chemistry, University of Jyväskylä, Jyväskylä, Finland
}

OPEN ACCESS

Edited by:

Kornelius Zeth,

Roskilde University, Denmark

Reviewed by:

Stephane Rety,

INSERM U1210 Laboratoire de

Biologie et Modelisation de la Cellule

(LBMC), France

Jean-David Rochaix,

Université de Genève, Switzerland

*Correspondence:

Ivan Gushchin

ivan.gushchin@phystech.edu

Specialty section:

This article was submitted to

Structural Biology

a section of the journal

Frontiers in Molecular Biosciences

Received: 10 July 2019

Accepted: 13 November 2019

Published: 29 November 2019

Citation:

Novitskaia O, Buslaev P and

Gushchin I (2019) Assembly of Spinach Chloroplast ATP Synthase

Rotor Ring Protein-Lipid Complex.

Front. Mol. Biosci. 6:135.

doi: 10.3389/fmolb.2019.00135
Rotor ATPases are large multisubunit membrane protein complexes found in all kingdoms of life. The membrane parts of these ATPases include a ring-like assembly, so-called c-ring, consisting of several subunits c, plugged by a patch of phospholipids. In this report, we use a nature-inspired approach to model the assembly of the spinach (Spinacia oleracea) $\mathrm{c}_{14}$ ring protein-lipid complex, where partially assembled oligomers are pulled toward each other using a biasing potential. The resulting assemblies contain 23 to 26 encapsulated plug lipids, general position of which corresponds well to experimental maps. However, best fit to experimental data is achieved with 15 to 17 lipids inside the c-ring. In all of the simulations, the lipids from one leaflet (loop side of the c subunit) are ordered and static, whereas the lipids from the other leaflet are disordered and dynamic. Spontaneous permeation of water molecules toward Glu61 at the active site is also observed. The presented assembly approach is expected to be generalizable to other protein complexes with encapsulated lipid patches.

Keywords: membrane protein, membrane insertion, complex assembly, annular lipids, protein-lipid interactions

\section{INTRODUCTION}

Rotor ATPases are large multisubunit membrane protein complexes found in all kingdoms of life that convert the energy stored in transmembrane potential into the energy of a covalent bond in adenosine triphosphate (ATP), or vice versa (Walker, 2013; Junge and Nelson, 2015). Generally, ATPases consist of two large parts, one of which is soluble and another one is embedded in the membrane. The membrane part includes a so-called c-ring-a symmetric homo- or heterooligomer of subunits $c$, which is rotating in the membrane during the protein operation (Figure 1A). Currently, c-rings consisting of 8 to 17 protomers are known (Kühlbrandt and Davies, 2016; Schulz et al., 2017). The number of $c$ subunits defines the energetics of ATP synthase because it corresponds to the number of ions $\left(\mathrm{H}^{+}\right.$or $\left.\mathrm{Na}^{+}\right)$transported per a full $360^{\circ}$ rotation, which invariably results in synthesis or hydrolysis of 3 ATP molecules. Most of the subunits c consist of two transmembrane helices with the connecting loop directed toward the soluble part of ATP synthase, whereas others have four transmembrane helices, which presumably results from gene duplication. Some of the c-rings can self-assemble (Arechaga et al., 2002) whereas others require accessory subunits such as UncI (Ozaki et al., 2008). The inside pore of the c-rings in some cases is plugged by phospholipids (Meier et al., 2001; Oberfeld et al., 2006).

Highly detailed atomistic structures of isolated c-rings and whole ATP synthases have recently became available (Kühlbrandt and Davies, 2016; Guo and Rubinstein, 2018; Murphy et al., 2019). High resolution crystallographic structures reveal both the overall fold as well as the geometry of the active site comprising a carboxylate amino acid, which is de- and re-protonated during 

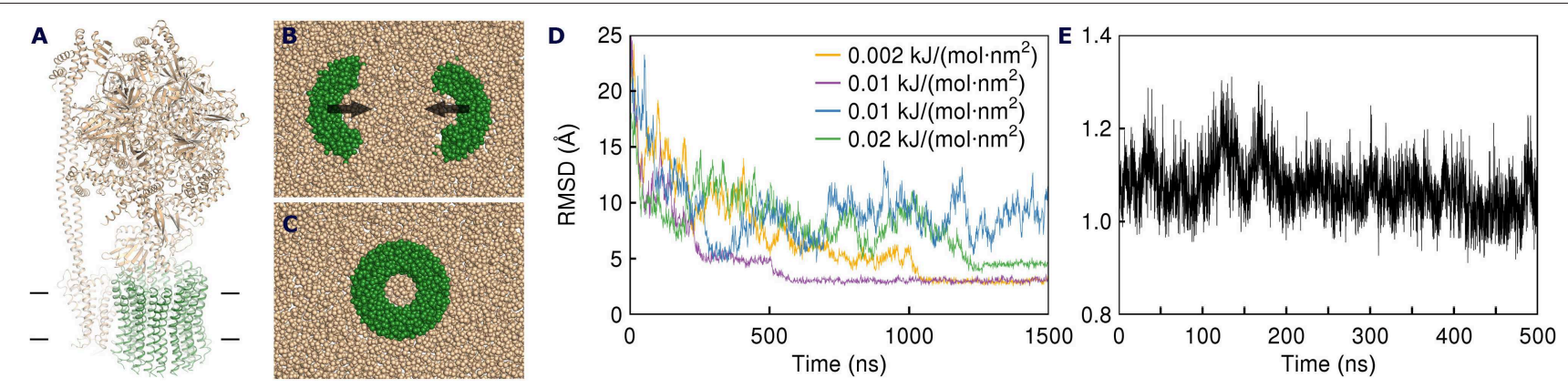

FIGURE 1 | Assembly of the spinach ATP synthase rotor ring protein-lipid complex. (A) Structure of the spinach ATP synthase (Hahn et al., 2018). The rotor ring is highlighted in green and the approximate membrane boundaries are shown with black lines. (B) The rotor ring assembly approach. $\mathrm{C}_{7}$ oligomers are separated by $5 \mathrm{~nm}$ and pulled toward the experimentally determined positions using harmonic restraints. (C) Assembled $\mathrm{C}_{14}$ ring. (D) Convergence of the $\mathrm{C}_{7}$ oligomers during pulling simulations. Yellow and magenta traces converge to $3 \AA$; the green simulation is trapped in a high-RMSD conformation (4.6 $\AA$ ); the blue simulation doesn't converge in $1,500 \mathrm{~ns}$. (E) RMSD of $\mathrm{C}_{\alpha}$ positions in unrestrained atomistic simulation of the assembled c-ring with 17 lipids inside relative to the starting experimental structure (PDB ID 6FKF)

the protein operation. Structures obtained using single particle cryo-electron microscopy (EM) currently have lesser resolution, but are better at revealing the overall complex structure, often in several conformations. Interestingly, in some recent EM structures of yeast V-ATPase, additional transmembrane (TM) helices have been observed inside the c-ring (Mazhab-Jafari et al., 2016; Roh et al., 2018). While the protein components of ATP synthases are generally well resolved in the structures, the densities for the surrounding lipid molecules, including those presumably trapped inside the c-ring, are of lesser quality. In the best available structure, that of Enterococcus hirae V-ATPase $\mathrm{K}_{10}$ ring, electron densities of 20 diacylglycerol/phosphatidylglycerol molecules are resolved in the immediate vicinity of the protein inside the ring, but not in its center or outside the ring (Murata et al., 2005). Most probably this is due to the great flexibility and dynamic nature of lipids (Buslaev et al., 2016) and lack of specific lipid-protein interactions, or due to detergent extraction used for sample preparation. Consequently, the identities of lipids, and their exact positions, are not well resolved in the available experimental density maps, and corresponding moieties are not modeled in experimental structures.

The lipids in and around c-rings were simulated in several previous studies, for example (Aksimentiev et al., 2004; Sengupta et al., 2009; Krah et al., 2010; Pogoryelov et al., 2010). In the smaller $c_{10}$ rings, usually, four lipids were modeled inside (Aksimentiev et al., 2004; Sengupta et al., 2009; Zhou et al., 2017a). Larger $c_{14}$ and $c_{15}$ rings were simulated with 12 and 15 lipids in the central plug, respectively (Krah et al., 2010). Finally, an even larger $\mathrm{K}_{10}$ ring (40 TM helices) should contain even more lipids (Murata et al., 2005; Leone et al., 2015). Molecular dynamics (MD) simulations have also been used to probe the specific interactions of cardiolipin with the outer surface of metazoan c-rings (Duncan et al., 2016).

\footnotetext{
Abbreviations: ATP, adenosine triphosphate; POPC, palmitoyl-oleoylphosphatidylcholine; DOPC, dioleoyl-phosphatidylcholine; DPPC, dipalmitoylphosphatidylcholine; EM, electron microscopy; MD, molecular dynamics; TM, transmembrane; RMSD, root-mean-square deviation; NC, N- and C-termini; AA, all atom; CG, coarse grained; RSCC, real space correlation coefficient.
}

Recently, a Cryo-EM structure of spinach chloroplast ATP synthase with recognizable densities in the membrane region has become available (Hahn et al., 2018), providing an exciting opportunity to compare the modeled lipid positions with the experimental data. Neither this structure, nor previously determined crystallographic structures of similar chloroplast $\mathrm{c}_{14}$ rings (Vollmar et al., 2009; Saroussi et al., 2012; Balakrishna et al., 2014), contain lipid molecules. Here, we use an approach inspired by the natural assembly process to model the lipids inside the c-ring. It builds upon the existing approaches for insertion of preformed oligomers (Kandt et al., 2007; Lindahl and Sansom, 2008; Wolf et al., 2010; Biggin and Bond, 2015; Javanainen and Martinez-Seara, 2016), and uses a biasing force to assemble the whole ring, essentially by incorporating experimental restraints into the simulation. Using the approach, we observed trapping of up to 26 palmitoyl-oleoyl-phosphatidylcholine (POPC) lipids inside the c-ring, and positions of these lipids correspond well to the experimentally determined density maps. Then, we compare simulations with different numbers of lipids inside the c-ring to experimental maps and find the best fitting ones.

\section{RESULTS AND DISCUSSION}

Our approach to assembly of the c-ring protein-lipid complex is inspired by the natural assembly process. In nature, probably, subunits $\mathrm{c}$ are synthesized by ribosome independently and inserted into the membrane one-by-one. After that, the protomers assemble into oligomers, sometimes observed in atomic force microscopy images (Müller et al., 2001; Pogoryelov et al., 2005), and eventually form complete rings. Some of the c-rings can assemble in liposomes in vitro while others require presence of accessory proteins (Rühle and Leister, 2015). On the contrary, the existing computational methods for insertion of membrane proteins into membranes involve quite unnatural assembly steps: most of the approaches artificially insert fully assembled proteins and protein complexes into pre-equilibrated membrane patches, whereas others rely on the self-assembly of the membrane around the membrane proteins or their complexes 
TABLE 1 | Details of the assembly simulations.

\begin{tabular}{|c|c|c|c|c|c|c|c|c|}
\hline Lipid & $\begin{array}{l}\text { Restraint } \\
\text { coefficient, } \\
\text { kJ/ } \\
\left(\mathrm{mol} \cdot \mathrm{nm}^{2}\right)\end{array}$ & $\begin{array}{c}\# \\
\text { assembled } \\
\text { (not } \\
\text { assembled) }\end{array}$ & $\begin{array}{l}\text { Percent } \\
\text { assembled }\end{array}$ & $\begin{array}{c}\text { Convergence time, } \\
\mu \mathrm{s}\end{array}$ & RMSD, Å & $\begin{array}{l}\text { \# of lipids, loop } \\
\text { side }\end{array}$ & $\begin{array}{c}\text { \# of lipids, NC } \\
\text { side }\end{array}$ & Total \# of lipids \\
\hline POPC & 0.002 & $1(4)$ & 20 & 1.1 & 3.0 & 10 & 13 & 23 \\
\hline POPC & 0.005 & $0(8)$ & 0 & n.d. & n.d. & n.d. & n.d. & n.d. \\
\hline POPC & 0.01 & $3(6)$ & 33 & $0.7,1.0,4.0$ & $3.1,3.1,4.7$ & $9,10,11$ & $14,14,15$ & $23,24,26$ \\
\hline POPC & 0.02 & $4(4)$ & 50 & $0.9,0.8,0.5,1.4$ & $4.6,4.5,3.0,4.5$ & $10,10,10,10$ & $15,14,14,13$ & $25,24,24,23$ \\
\hline DOPC & 0.01 & $5(8)$ & 38 & $0.9,1.4,1.2,1.5,0.6$ & $4.7,4.6,4.5,4.6,4.5$ & $10,9,9,10,9$ & $15,14,13,15,13$ & $25,23,22,24,22$ \\
\hline DPPC & 0.01 & $5(10)$ & 33 & $1.0,1.4,2.2,0.8,0.6$ & $4.5,3.0,4.5,3.0,3.4$ & $11,10,10,10,10$ & $14,14,15,14,14$ & $25,24,25,24,24$ \\
\hline
\end{tabular}

Convergence times, RMSD and numbers of lipids are indicated only for the trajectories where complete assembly has been observed.
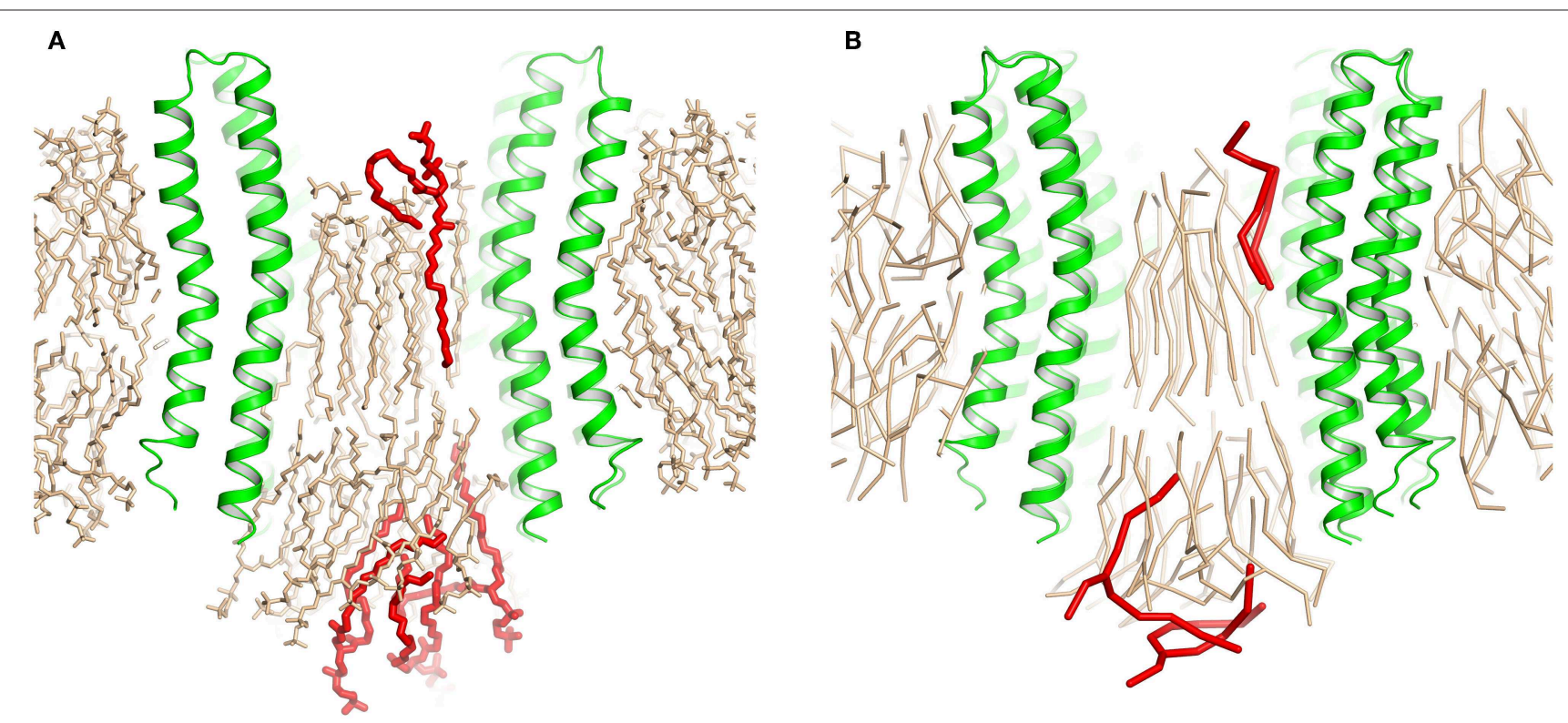

FIGURE 2 | Extrusion of excess lipids from the c-ring. Position of the lipids that are pushed out (red) is shown relative to the position of other lipids (beige). (A) Atomistic simulation with 25 lipids. (B) Coarse grained simulation with 23 lipids.

(Kandt et al., 2007; Lindahl and Sansom, 2008; Wolf et al., 2010; Biggin and Bond, 2015; Javanainen and Martinez-Seara, 2016). In most cases, these unnatural approaches work very well, but in special cases, where some of the lipids are encapsulated within the membrane protein or protein complex, special care may be required.

Since the c subunits are relatively simple hairpin-shaped proteins, and there are no lipid molecules trapped in the c:c interfaces, we skipped the initial oligomerization steps in our simulations and started directly from partially assembled rings. Partially assembled rings have been simulated before, when the true stoichiometry of the ring was not known (Schlegel et al., 2012) or in a permeability study (Zhou et al., 2017b). Spinach c-ring contains 14 protomers, which we separated into two heptamers for simplicity of the assembly protocol. While it is likely that in nature the assembly proceeds via oligomers of different stoichiometry, we believe that the resulting arrangements of encapsulated lipids should be similar for different assembly pathways. The $c_{7}$ heptamers, separated by $5 \mathrm{~nm}$, were inserted into the membranes consisting of the commonly used model lipid POPC and were pulled toward the experimental positions by a biasing potential realized as a harmonic restraint to the experimental structure (Figures 1B,C). While theoretically the halves could assemble into a complete rotor without any biasing force, in practice this might require a large amount of computational time. Using a force too weak might also result in slow assembly. On the contrary, applying a force too strong could result in unnatural deformation of the bilayer and trapping of a system in unnatural conformation. Therefore, we tested several harmonic biasing potentials with spring constants of $0.002,0.005,0.01$, and $0.02 \mathrm{~kJ} /\left(\mathrm{mol} \cdot \mathrm{nm}^{2}\right)$ and followed root-mean-square deviation (RMSD) of atomic positions relative to the reference experimental structure (Hahn et al., 2018). Using a coarse grained (CG) force field allowed for much faster assembly simulations, due to both computational efficiency and faster dynamics in CG simulations (Marrink 


\section{A}

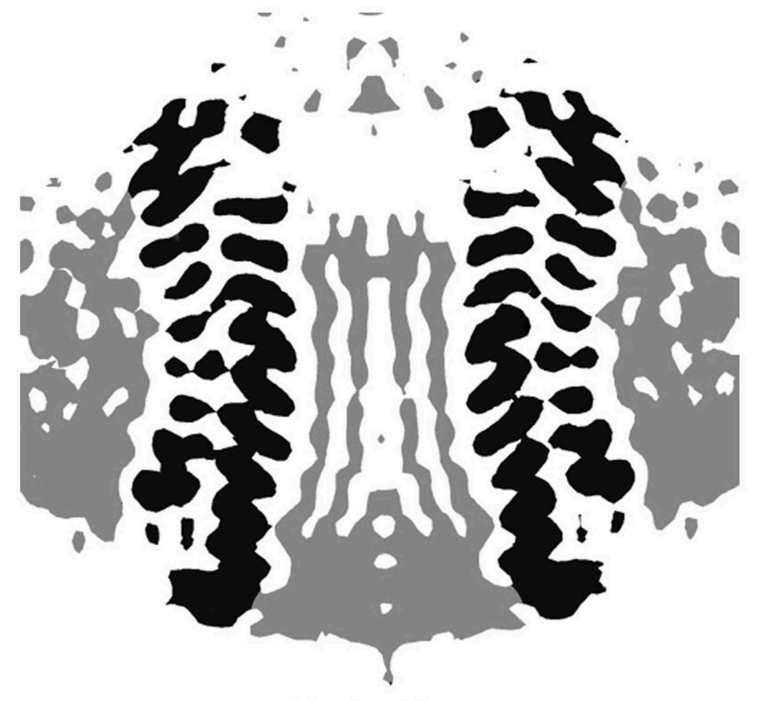

Experimental map
B

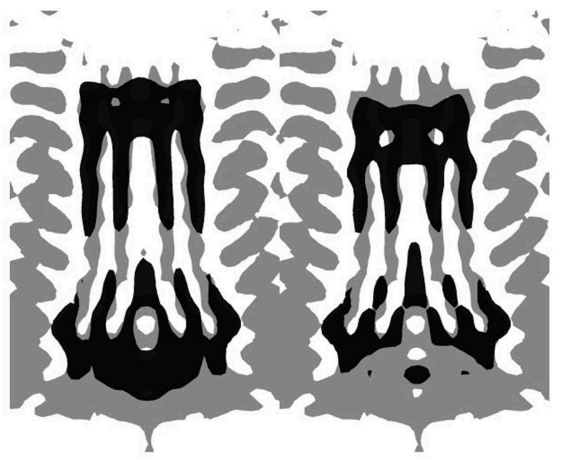

C
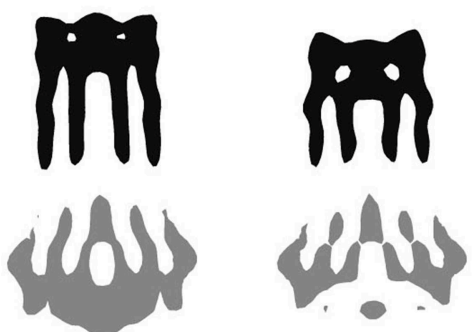

$6+9$ lipids

atomistic

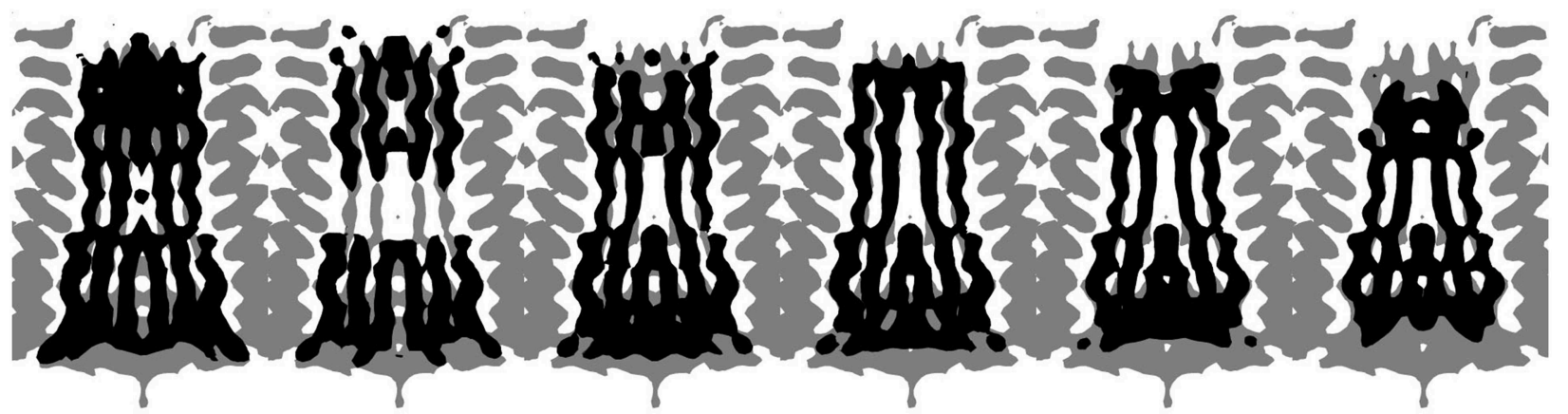

E

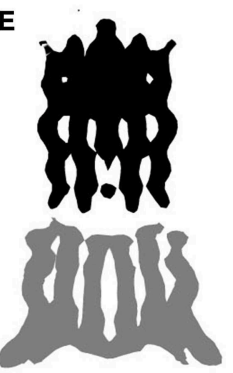

$10+13$ lipids

coarse-grained
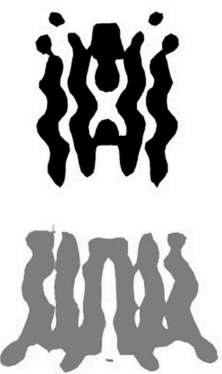

9+12 lipids

coarse-grained

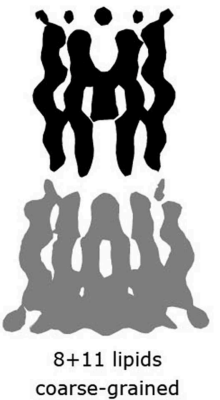

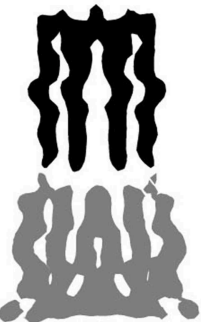

$7+10$ lipids

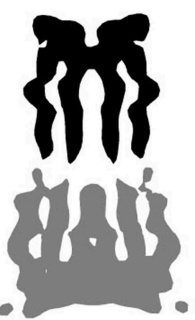

$6+9$ lipids

coarse-grained

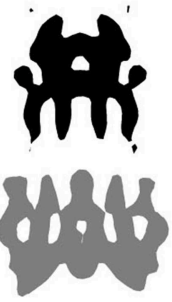

$5+8$ lipids coarse-grained

FIGURE 3 | Comparison of experimental and simulated lipid densities. In all panels, the protein positions are aligned. Experimental map is shown at the level of 0.2 a.u., CG and atomistic simulated maps are shown at the level of 1.3 and 7 a.u., respectively. (A) 14 -fold averaged Cryo-EM map. Densities corresponding to subunits $\mathrm{c}$ are shown in black, other densities are shown in gray. (B) Comparison of the results of atomistic simulations with the experimental map. Experimental map is shown in gray, simulated densities are shown in black. (C) Densities corresponding to lipids at the loop side (black) and to lipids at the NC-side (gray).

(D) Comparison of the results of coarse grained simulations with the experimental map. Experimental map is shown in gray, simulated densities are shown in black. (E) Densities corresponding to lipids at the loop side (black) and to lipids at the NC-side (gray).

and Tieleman, 2013; Buslaev and Gushchin, 2017). In many of the simulations, the structures converged into an assembled ring in $<1.5 \mu \mathrm{s}$. The numbers of lipids trapped at the loop side and the $\mathrm{N}$ - and C-termini (NC)-side were consistent in different simulation runs: 9 to 11 and 13 to 15 , respectively (Table 1). Using stronger restraints often resulted in trapping of 
the complex in a high-RMSD conformation $(\sim 4.6 \AA)$, whereas using weaker restraints resulted in most cases in absence of convergence (Figure 1D and Table 1).

After the heptamers have approached each other, we followed the initial weak restraint simulation step by a series of three consecutive 200 ps simulations with strong restraint spring constants of 10,100 , and $1,000 \mathrm{~kJ} /\left(\mathrm{mol} \cdot \mathrm{nm}^{2}\right)$, after which the RMSD relative to the experimental structure dropped below 0.8 $\AA$. After that, the CG positions of plug lipids were converted into all atom (AA) representation using backward (Wassenaar et al., 2014), and the CG protein model was replaced by the experimental atomic structure. The resulting complex was solvated by water molecules and simulated without restrains. First, we conducted an AA simulation with an average amount of plug lipids (25). The c-ring remained stable but some lipids

TABLE 2 | Real space correlation coefficients (RSCC) for simulations with different numbers of lipids.

\begin{tabular}{|c|c|c|c|c|c|c|c|c|}
\hline \multirow[b]{2}{*}{ Region } & \multicolumn{8}{|c|}{ System } \\
\hline & $\begin{array}{l}\text { 7+10 } \\
\text { lipids } \\
\text { atomistic }\end{array}$ & $\begin{array}{c}6+9 \\
\text { lipids } \\
\text { atomistic }\end{array}$ & $\begin{array}{l}10+13 \\
\text { lipids } \\
\text { CG }\end{array}$ & $\begin{array}{c}9+12 \\
\text { lipids } \\
\text { CG }\end{array}$ & $\begin{array}{c}8+11 \\
\text { lipids } \\
\text { CG }\end{array}$ & $\begin{array}{c}7+10 \\
\text { lipids } \\
\text { CG }\end{array}$ & $\begin{array}{c}6+9 \\
\text { lipids } \\
\text { CG }\end{array}$ & $\begin{array}{l}5+8 \\
\text { lipids } \\
\text { CG }\end{array}$ \\
\hline Overall & 0.78 & 0.78 & 0.60 & 0.58 & 0.62 & 0.71 & 0.75 & 0.71 \\
\hline Loop side & 0.7 & 0.69 & 0.40 & 0.36 & 0.40 & 0.59 & 0.66 & 0.59 \\
\hline NC side & 0.87 & 0.91 & 0.77 & 0.79 & 0.80 & 0.81 & 0.83 & 0.83 \\
\hline
\end{tabular}

from each side of the ring were pushed out of the c-ring core (Figure 2A). Therefore, we have attempted a second simulation, starting from the converged structure with 23 plug lipids. During the strong restraint preparatory CG simulation, three lipids were also pushed out of the c-ring (Figure 2B).

These short simulations suggested that our assembly simulations overestimated the number of lipids inside the spinach chloroplast c-ring, and prompted us to conduct a quantitative comparison of the lipid positions obtained in simulations to experiment. The original structure (Hahn et al., 2018) is not symmetrical due to presence of ATP synthase subunits other than the c-ring. Yet, the ring itself in the experimental structure is symmetric, and a free c-ring in a lipid membrane is expected to be symmetric as well. Thus, for analysis, we averaged the experimental Cryo-EM map according to the 14-fold symmetry of the ring (Figure 3 ). We have conducted a number of $1 \mu$ s long CG simulations of c-ring assemblies where lipids were removed from each side one-by-one, while the protein particles were restrained to the experimental positions. The densities obtained in simulations were averaged over the trajectory length and also over the 14-fold symmetry of the ring. The best fit is observed visually for the system with 6 or 7 lipids at the loop side and 9 or 10 lipids at the NC side (Figure 3D). Real space correlation coefficients (RSCC), used routinely to compare EM maps to each other and to evaluate model-density fits (Afonine et al., 2018), highlight the 6:9 system as the best fitting one with the RSCC value of $\sim 0.75$ (Table 2).
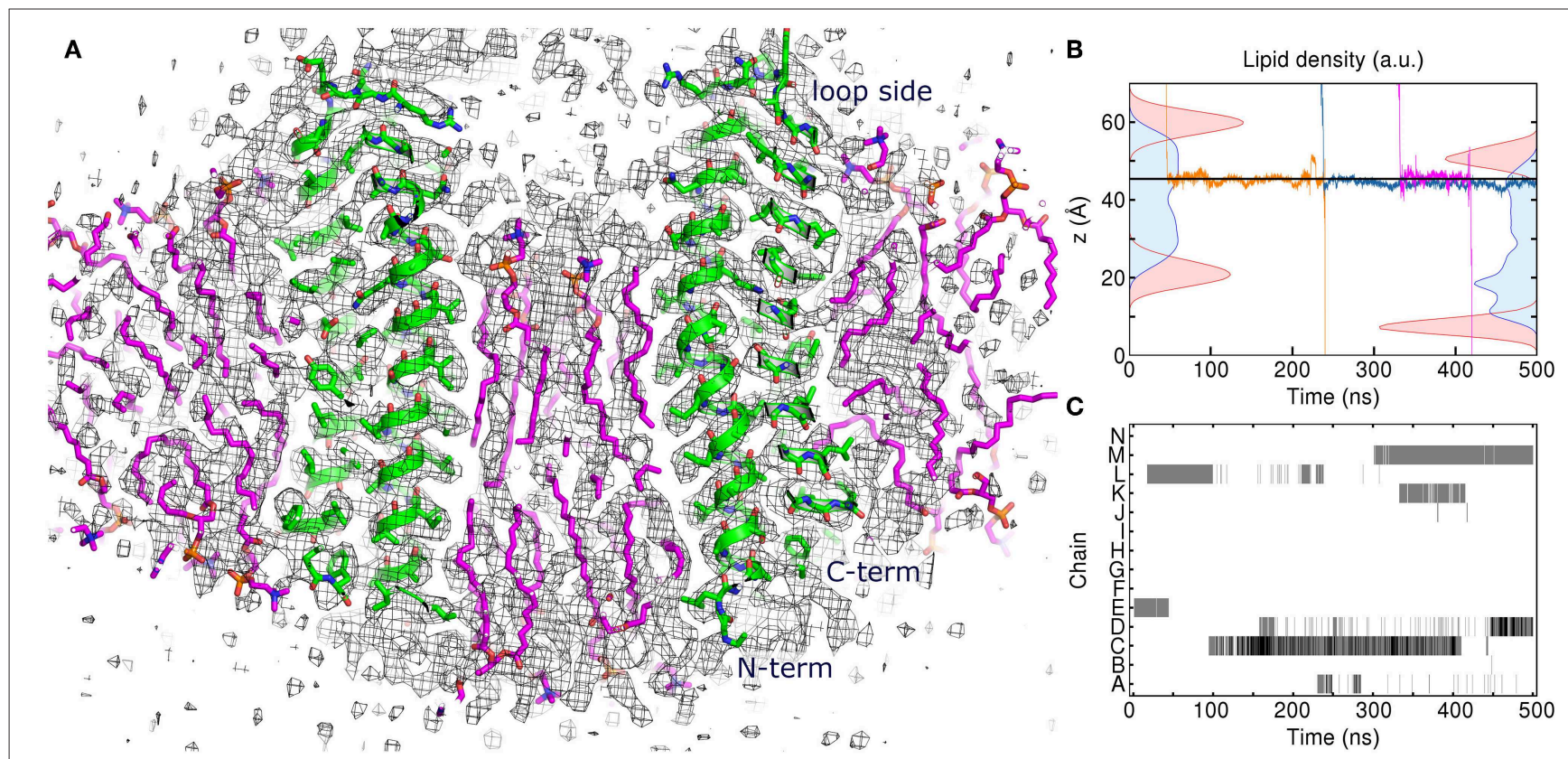

FIGURE 4 | Atomistic simulation of the spinach chloroplast ATP synthase rotor ring. (A) Comparison of the assembled model with 17 lipids inside the c-ring at 250 ns with the experimental Cryo-EM map EMD_4273 (Hahn et al., 2018) contoured at the level of 3 $\sigma$ (black). Excellent correspondence between positions of protein atoms (green) and experimental map is observed. The lipids (magenta) fit very well into the densities in the membrane region. (B) Density distributions of outer (left) and inner (right) POPC phosphate (red) and diacylglycerol (blue) moieties overlaid with three exemplary trajectories of permeating water molecules. Average position of Glu61 centers of mass is shown using the solid line. (C) Contact maps between water molecules and Glu61 side chains. Presence of water molecules whose oxygen atoms are within $4 \AA$ of Glu61 $\mathrm{O}_{\varepsilon 1}$ and $\mathrm{O}_{\varepsilon 2}$ atoms is indicated using gray (1 molecule) and black (2 molecules). 
The molecular entities with RSCC values above 0.7 are generally recognized as consistent with corresponding maps (Neumann et al., 2018). Overall, the 6:9 system reproduces the densities in the acyl chain region very well, but clearly lacks needed density in the head group region.

After the CG simulations, we have also conducted short atomistic simulations of $6: 9$ and 7:10 systems. Both revealed similar correlation with the experimental map (Table 2), with the 6:9 system severely lacking densities in the head group region, and the 7:10 system having better correspondence there (Figure 3B). The reason for the discrepancy in the head group region might be that we used the model lipid POPC in our simulations, whereas the natural thylakoid membranes contain a complex mixture of phosphatidylglycerol, monogalactosyldiacylglycerol, digalactosyldiacylglycerol and sulfoquinovosyldiacylglycerol lipids (Webb and Green, 1991). Phosphate and choline moieties of POPC have joint molecular weight of about $200 \mathrm{Da}$, whereas one galactose moiety weighs around $180 \mathrm{Da}$, two galactoses weigh $\sim 360 \mathrm{Da}$, and sulfoquinovose weighs $\sim 250 \mathrm{Da}$. Therefore, native lipids have head groups with higher molecular mass and bigger volume, and this might account for the additional density observed in the lipid head group region of experimentally determined density maps.

To gain insight into atomistic details and to probe the system stability, we have simulated the 7:10 system without any applied restraints for $500 \mathrm{~ns}$. Overall, the system was stable with the protein $\mathrm{C}_{\alpha}$ RMSD of $\sim 1.1 \AA$ (Figure 1E) and the lipids remaining close to the original position. We observe a reasonable correspondence of modeled lipid positions to the experimental densities (Figure 4A). Interestingly, the lipids encapsulated within the $\mathrm{c}$-ring are displaced relative to the surrounding bilayer (Figure 4B), as it was observed before in experimental and modeled structures of other c-rings (Murata et al., 2005; Krah et al., 2010). At the loop side of the c subunit, the lipids are straightened and immobile. At the NC side, the lipids are more disordered and dynamic, forming a conical shape. Oleoyl chains of three POPC molecules at the NC side are bent so that some acyl chains are oriented along the membrane plane and perpendicular to other palmitoyl and oleoyl moieties. Finally, while no water molecules were initially placed within the membrane, we observed diffusion of several molecules into the membrane toward the active site glutamate (Figures 4B,C).

Based on the reported simulations and comparison of density distributions in the acyl chain region to the experimental data (Figure 3 and Table 2), we conclude that the experimental maps are best described by systems with 15 to 17 lipid molecules. These numbers are in contrast with 23 to 26 lipids observed in biasing potential-driven assembly simulations. Is it possible that trapping of the excessive number of lipids is a consequence of using a biasing potential? Our analyses indicate that this likely might not the case. First, most of the assemblies with the biasing potential coefficient below $0.01 \mathrm{~kJ} /\left(\mathrm{mol} \cdot \mathrm{nm}^{2}\right)$ do not converge in reasonable time (Table 1), and thus the biasing potentials are not too excessive and affect the system moderately. Second, some lipids are known to interact tightly

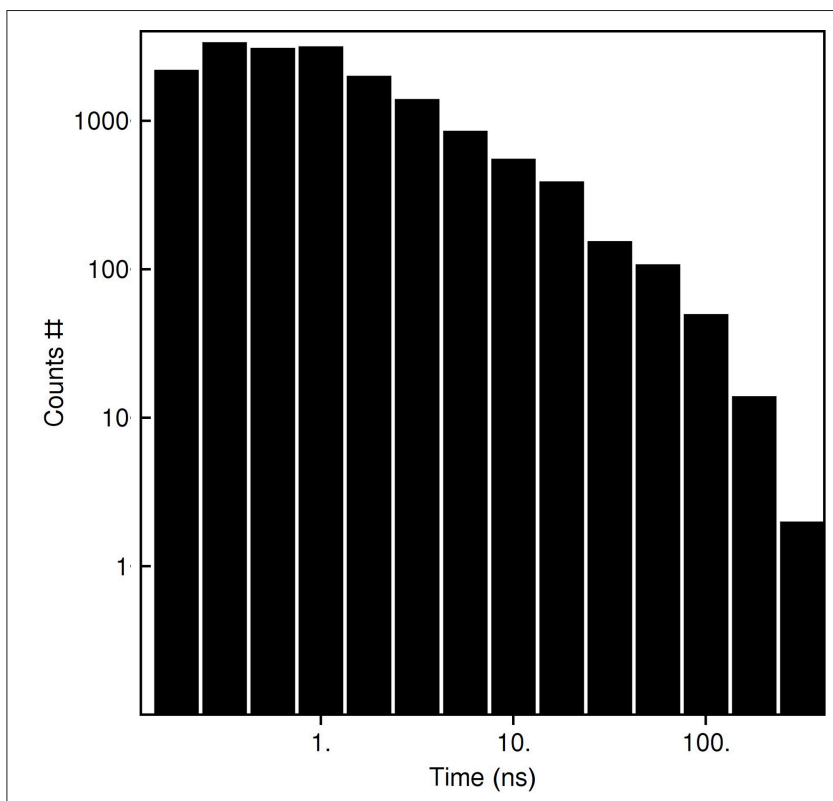

FIGURE 5 | Residence times of POPC lipids in the $c_{7}$ inner volume defined by the outermost protomers of the half-rings.

with corresponding membrane proteins, and such interaction might prevent reaching equilibrium number of lipids inside the c-ring in accelerated assembly simulation. However, analysis of the residence times of POPC lipids in the inner volume of $\mathrm{c}_{7}$ half-rings separated by $5 \mathrm{~nm}$ reveals that lipids in most cases do not stay within the cavity for longer than $\sim 100$ ns (Figure 5). Given that the typical assembly takes $0.5-1.5 \mu \mathrm{s}$, the lipids have an opportunity to leave the cavity during the assembly simulation. Third, lipids are known to co-diffuse with membrane proteins (Niemelä et al., 2010), and thus might be dragged by the half-rings pulled by the biasing potential. In assembly simulations with biasing potential coefficients of 0.01 $\mathrm{kJ} /\left(\mathrm{mol} \cdot \mathrm{nm}^{2}\right)$ most of the lipids that are eventually trapped inside the c-ring are initially positioned in between the $c_{7}$ halfrings (Figure 6A). On the contrary, in the $0.002 \mathrm{~kJ} /\left(\mathrm{mol} \cdot \mathrm{nm}^{2}\right.$ ) assembly simulation, the lipids are evidently free to diffuse inside and outside the space between the approaching half-rings, and most of the lipids that are eventually encapsulated are initially positioned outside of the starting positions of $\mathrm{c}_{7}$ half-rings (Figure 6B), yet still 23 lipids are trapped. Finally, assembly simulations with dioleoyl-phosphatidylcholine (DOPC), a lipid with two unsaturated, and strongly disordered, oleoyl chains, and dipalmitoyl-phosphatidylcholine (DPPC), a lipid with two saturated, and ordered, acyl chains result in very similar numbers of trapped lipids (Table 1). To sum up, it appears that the effects arising due to using a biasing potential, if they are present, are moderate and do not explain the difference between the numbers of 23-26 lipids trapped in the assembly simulations and 15-17 lipids observed to fit best the experimental maps. Therefore, it is tempting to speculate that during the natural assembly process the c-ring might initially include a 


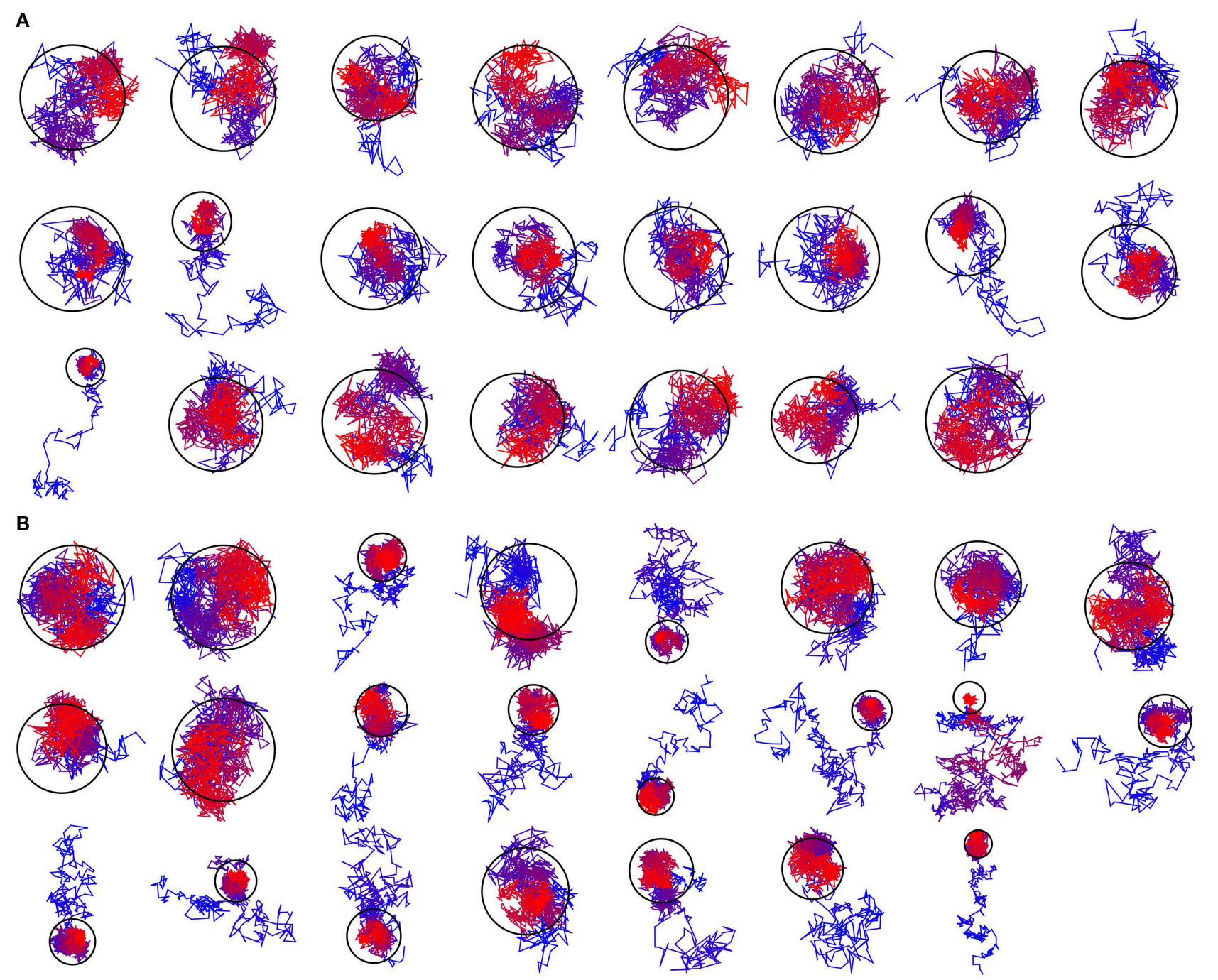

FIGURE 6 | Exemplary trajectories of lipids that are eventually encapsulated inside the c-ring. The color changes gradually from blue for the starting position to red for the final position. The ring represents the assembled c-ring. (A) Trajectory assembled using the biasing potential coefficient of $0.01 \mathrm{~kJ} /\left(\mathrm{mol}^{\prime} \mathrm{nm}{ }^{2}\right)$. Most of the encapsulated lipids are initially positioned in between the $\mathrm{c}_{7}$ half-rings. (B) Trajectory assembled using the biasing potential coefficient of $0.002 \mathrm{~kJ} /\left(\mathrm{mol} \cdot \mathrm{nm}^{2}\right.$ ). Most of the encapsulated lipids are initially positioned outside of the starting positions of $\mathrm{C}_{7}$ half-rings.

larger number of lipids, and then consequently some lipids are extruded, as depicted in Figure 2, and either can diffuse away themselves or might be carried away by some soluble transport proteins.

Preliminary data shows that the presented approach works well also for larger $\mathrm{K}_{10}$ (40 TM helices) and smaller $\mathrm{c}_{13}$ (26 TM helices) rings (data not shown). We believe that it can be used for assembly of other protein complexes with encapsulated lipids such as the multidrug exporter AcrB (Qiu et al., 2018) or oligomeric light-driven pumps, microbial rhodopsins (Gushchin and Gordeliy, 2018; Bratanov et al., 2019; Kovalev et al., 2019) as well. As lipids are often critical for correct folding and operation of membrane proteins (Cournia et al., 2015; Hedger and Sansom, 2016; Gupta et al., 2017), reproducing their correct positions in simulations is of utmost importance.

\section{METHODS}

\section{Preparation of Starting Models}

Starting coordinates for the spinach c-ring were taken from PDB ID 6FKF. The symmetry axis of the ring was oriented along the $\mathrm{z}$ axis. For assembly simulations, half-ring oligomers were separated by $5 \mathrm{~nm}$ along the $\mathrm{x}$ axis. Atomic structures were converted into Martini representation using martinize.py (http://cgmartini.nl/index.php/tools2/proteins-and-bilayers/ 204-martinize). CG lipids, ions and water particles were added using insane (Wassenaar et al., 2015). The resulting CG systems contained 1308 POPC molecules and 8, 15 and $25776 \mathrm{Na}^{+}, \mathrm{Cl}^{-}$ and water particles, respectively, in a rectangular unit cell with dimensions of $\sim 24 \times 18 \times 12 \mathrm{~nm}^{3}$. For atomistic simulations, the systems were reassembled using the internal lipid positions 
taken from CG assembly simulations (Wassenaar et al., 2014), the experimental c-ring structure, and the external lipid positions generated using CHARMM-GUI (Jo et al., 2007). The resulting AA system contained 17 lipids inside the c-ring, 242 lipids outside of the c-ring, and $7 \mathrm{Cl}^{-}$and 28348 water molecules, in a hexagonal unit cell with dimensions of $\sim 11.5 \times 11.5 \times 13$ $\mathrm{nm}^{3}$. All simulations were performed using periodic boundary conditions. pKa values of titratable amino acids were determined using PROPKA3 (Olsson et al., 2011). Active site Glu61 were protonated. Glu37 of even chains were protonated because of borderline $\mathrm{pKa}$ value of $\sim 6.8$. Other titratable amino acids and $\mathrm{N}$ - and $\mathrm{C}$ - termini were charged. All simulations were performed using GROMACS 5.1 (Abraham et al., 2015).

\section{General Details of the Simulations}

CG and AA simulations were conducted using leapfrog integrator with time steps of 20 and $2 \mathrm{fs}$, at a reference temperature of 350 and $303 \mathrm{~K}$, respectively, and at a reference pressure of 1 bar. Protein, lipid and water molecules were coupled to the temperature bath separately. Temperature was coupled using velocity rescale (Bussi et al., 2007) and Nosé-Hoover (Nosé, 1984) thermostats with coupling constant of $1 \mathrm{ps}^{-1}$, respectively. Pressure was coupled with semiisotropic ParrinelloRahman barostat (Parrinello and Rahman, 1981) with relaxation time of 12 or 5 ps, respectively.

\section{Details of CG Simulations}

CG simulations were conducted using Martini 2.2 (Marrink and Tieleman, 2013) and ELNEDYN (Periole et al., 2009) force fields. The center of mass of the reference structure was scaled with the scaling matrix of the pressure coupling. The non-bonded pair list was updated every 10 steps with cutoff of $1.1 \mathrm{~nm}$. Potentials shifted to zero at the cutoff of $1.1 \mathrm{~nm}$ and a reactionfield potential with $\varepsilon_{\mathrm{rf}}=\infty$ were used for treatment of the van der Waals and electrostatics interaction as recommended (de Jong et al., 2016).

\section{Details of AA Simulations}

AA simulations were conducted using CHARMM36 (Klauda et al., 2010; Best et al., 2012) force field. The covalent bonds to

\section{REFERENCES}

Abraham, M. J., Murtola, T., Schulz, R., Páll, S., Smith, J. C., Hess, B., et al. (2015). GROMACS: high performance molecular simulations through multilevel parallelism from laptops to supercomputers. SoftwareX 1-2, 19-25. doi: 10.1016/j.softx.2015.06.001

Afonine, P. V., Klaholz, B. P., Moriarty, N. W., Poon, B. K., Sobolev, O. V., Terwilliger, T. C., et al. (2018). New tools for the analysis and validation of cryo-EM maps and atomic models. Acta Crystallogr D Struct. Biol. 74, 814-840. doi: $10.1107 /$ S2059798318009324

Aksimentiev, A., Balabin, I. A., Fillingame, R. H., and Schulten, K. (2004). Insights into the molecular mechanism of rotation in the Fo sector of ATP synthase. Biophys. J. 86, 1332-1344. doi: 10.1016/S0006-3495(04)74205-8

Arechaga, I., Butler, P. J. G., and Walker, J. E. (2002). Self-assembly of ATP synthase subunit c rings. FEBS Lett. 515, 189-193. doi: 10.1016/S0014-5793(02) 02447-X hydrogen were constrained using SHAKE algorithm (Ryckaert et al., 1977). The non-bonded pair list was updated every 20 steps with cutoff of $1.2 \mathrm{~nm}$. Force-based switching function with the switching range of $1.0-1.2 \mathrm{~nm}$ and particle mesh Ewald (PME) method with $0.12 \mathrm{~nm}$ Fourier grid spacing and $1.2 \mathrm{~nm}$ cutoff were used for treatment of the van der Waals and electrostatics interactions.

\section{Details of RSCC Calculations}

RSCC values have been calculated using Chimera (Pettersen et al., 2004). The experimental map sampling density is $1.053 \AA$ in all directions, as used in the original report (Hahn et al., 2018). The simulated maps were obtained using the volmap utility in VMD (Humphrey et al., 1996) with the sampling density of $1 \AA$. The symmetry axes were determined using Chimera, and the maps were averaged with their symmetry-related images rotated by increments of $360^{\circ} / 14 \approx 25.7^{\circ}$. The map regions where the density of the simulated maps was above 0.8 (the volume inside the $c$ ring) have been used for RSCC calculation. For reference, the maps shown in Figures 3D,E are drawn at the level of 1.3.

\section{DATA AVAILABILITY STATEMENT}

The datasets generated for this study are available on request to the corresponding author.

\section{AUTHOR CONTRIBUTIONS}

$\mathrm{ON}$ and $\mathrm{PB}$ conducted the molecular dynamics simulations, analyzed the results and helped with manuscript preparation. IG designed and supervised the project, analyzed the results, and prepared the manuscript.

\section{FUNDING}

This study was funded by the Russian Foundation for Basic Research according to the research project No. 18-34-00986. We are grateful to the Center for Scientific Computing (CSC-IT Center for Science, Espoo, Finland) for computational resources.

Balakrishna, A. M., Seelert, H., Marx, S.-H., Dencher, N. A., and Grüber, G. (2014). Crystallographic structure of the turbine C-ring from spinach chloroplast F-ATP synthase. Biosci. Rep. 34:e00102. doi: 10.1042/BSR20130114

Best, R. B., Zhu, X., Shim, J., Lopes, P. E. M., Mittal, J., Feig, M., et al. (2012). Optimization of the additive CHARMM all-atom protein force field targeting improved sampling of the backbone $\phi, \psi$ and Side-Chain $\chi 1$ and $\chi 2$ dihedral angles. J. Chem. Theory Comput. 8, 3257-3273. doi: 10.1021/ct300400x

Biggin, P. C., and Bond, P. J. (2015). Molecular dynamics simulations of membrane proteins. Methods Mol. Biol. 1215, 91-108. doi: 10.1007/978-1-4939-1465-4_5

Bratanov, D., Kovalev, K., Machtens, J.-P., Astashkin, R., Chizhov, I., Soloviov, D., et al. (2019). Unique structure and function of viral rhodopsins. Nat. Commun. 10:4939. doi: 10.1038/s41467-019-12718-0

Buslaev, P., Gordeliy, V., Grudinin, S., and Gushchin, I. (2016). Principal component analysis of lipid molecule conformational changes in molecular dynamics simulations. J. Chem. Theory Comput. 12, 1019-1028. doi: $10.1021 /$ acs.jctc. 5 b01106 
Buslaev, P., and Gushchin, I. (2017). Effects of coarse graining and saturation of hydrocarbon chains on structure and dynamics of simulated lipid molecules. Sci. Rep. 7:11476. doi: 10.1038/s41598-017-11761-5

Bussi, G., Donadio, D., and Parrinello, M. (2007). Canonical sampling through velocity rescaling. J. Chem. Phys. 126:014101. doi: 10.1063/ 1.2408420

Cournia, Z., Allen, T. W., Andricioaei, I., Antonny, B., Baum, D., Brannigan, G., et al. (2015). Membrane protein structure, function, and dynamics: a perspective from experiments and theory. J. Membr. Biol. 248, 611-640. doi: $10.1007 / \mathrm{s} 00232-015-9802-0$

de Jong, D. H., Baoukina, S., Ingólfsson, H. I., and Marrink, S. J. (2016). Martini straight: boosting performance using a shorter cutoff and GPUs. Comput. Phys. Commun. 199, 1-7. doi: 10.1016/j.cpc.2015.09.014

Duncan, A. L., Robinson, A. J., and Walker, J. E. (2016). Cardiolipin binds selectively but transiently to conserved lysine residues in the rotor of metazoan ATP synthases. PNAS 113, 8687-8692. doi: 10.1073/pnas.16083 96113

Guo, H., and Rubinstein, J. L. (2018). Cryo-EM of ATP synthases. Curr. Opin. Struct. Biol. 52, 71-79. doi: 10.1016/j.sbi.2018.08.005

Gupta, K., Donlan, J. A. C., Hopper, J. T. S., Uzdavinys, P., Landreh, M., Struwe, W. B., et al. (2017). The role of interfacial lipids in stabilizing membrane protein oligomers. Nature 541, 421-424. doi: 10.1038/nature20820

Gushchin, I., and Gordeliy, V. (2018). Microbial rhodopsins. Subcell. Biochem. 87, 19-56. doi: 10.1007/978-981-10-7757-9_2

Hahn, A., Vonck, J., Mills, D. J., Meier, T., and Kühlbrandt, W. (2018). Structure, mechanism, and regulation of the chloroplast ATP synthase. Science 360:eaat4318. doi: $10.1126 /$ science.aat 4318

Hedger, G., and Sansom, M. S. P. (2016). Lipid interaction sites on channels, transporters and receptors: recent insights from molecular dynamics simulations. Biochim. et Biophys. Acta Biomembranes 1858, 2390-2400. doi: 10.1016/j.bbamem.2016.02.037

Humphrey, W., Dalke, A., and Schulten, K. (1996). VMD visual molecular dynamics. J. Mol. Grap. 14, 33-38. doi: 10.1016/0263-7855(96)00018-5

Javanainen, M., and Martinez-Seara, H. (2016). Efficient preparation and analysis of membrane and membrane protein systems. Biochim. Biophys. Acta 1858, 2468-2482. doi: 10.1016/j.bbamem.2016.02.036

Jo, S., Kim, T., and Im, W. (2007). Automated builder and database of protein/membrane complexes for molecular dynamics simulations. PLoS ONE 2:e880. doi: 10.1371/journal.pone.0000880

Junge, W., and Nelson, N. (2015). ATP synthase. Annu. Rev. Biochem. 84, 631-657. doi: 10.1146/annurev-biochem-060614-034124

Kandt, C., Ash, W. L., and Tieleman, D. P. (2007). Setting up and running molecular dynamics simulations of membrane proteins. Methods 41, 475-488. doi: 10.1016/j.ymeth.2006.08.006

Klauda, J. B., Venable, R. M., Freites, J. A., O'Connor, J. W., Tobias, D. J., Mondragon-Ramirez, C., et al. (2010). Update of the CHARMM all-atom additive force field for lipids: validation on six lipid types. J. Phys. Chem. B 114, 7830-7843. doi: 10.1021/jp101759q

Kovalev, K., Polovinkin, V., Gushchin, I., Alekseev, A., Shevchenko, V., Borshchevskiy, V., et al. (2019). Structure and mechanisms of sodium-pumping KR2 rhodopsin. Sci. Adv. 5:eaav2671. doi: 10.1126/sciadv.aav2671

Krah, A., Pogoryelov, D., Meier, T., and Faraldo-Gómez, J. D. (2010). On the structure of the proton-binding site in the $\mathrm{F}(\mathrm{o})$ rotor of chloroplast ATP synthases. J. Mol. Biol. 395, 20-27. doi: 10.1016/j.jmb.2009.10.059

Kühlbrandt, W., and Davies, K. M. (2016). Rotary ATPases: a new twist to an ancient machine. Trends Biochem. Sci. 41, 106-116. doi: 10.1016/j.tibs.2015.10.006

Leone, V., Pogoryelov, D., Meier, T., and Faraldo-Gómez, J. D. (2015). On the principle of ion selectivity in $\mathrm{Na}+\mathrm{H}+$-coupled membrane proteins: experimental and theoretical studies of an ATP synthase rotor. Proc. Natl. Acad. Sci. U.S.A. 112, E1057-1066. doi: 10.1073/pnas. 1421202112

Lindahl, E., and Sansom, M. S. P. (2008). Membrane proteins: molecular dynamics simulations. Curr. Opin. Struct. Biol. 18, 425-431. doi: 10.1016/j.sbi.2008.02.003

Marrink, S. J., and Tieleman, D. P. (2013). Perspective on the Martini model. Chem. Soc. Rev. 42, 6801-6822. doi: 10.1039/c3cs60093a

Mazhab-Jafari, M. T., Rohou, A., Schmidt, C., Bueler, S. A., Benlekbir, S., Robinson, C. V., et al. (2016). Atomic model for the membrane-embedded VO motor of a eukaryotic V-ATPase. Nature 539, 118-122. doi: 10.1038/nature19828
Meier, T., Matthey, U., Henzen, F., Dimroth, P., and Müller, D. J. (2001). The central plug in the reconstituted undecameric $c$ cylinder of a bacterial ATP synthase consists of phospholipids. FEBS Lett. 505, 353-356 doi: 10.1016/S0014-5793(01)02837-X

Müller, D. J., Dencher, N. A., Meier, T., Dimroth, P., Suda, K., Stahlberg, H., et al. (2001). ATP synthase: constrained stoichiometry of the transmembrane rotor. FEBS Lett. 504, 219-222. doi: 10.1016/S0014-5793(01)02708-9

Murata, T., Yamato, I., Kakinuma, Y., Leslie, A. G. W., and Walker, J. E. (2005). Structure of the rotor of the V-Type Na+-ATPase from Enterococcus hirae. Science 308, 654-659. doi: 10.1126/science.1110064

Murphy, B. J., Klusch, N., Langer, J., Mills, D. J., Yildiz, Ö., and Kühlbrandt, W. (2019). Rotary substates of mitochondrial ATP synthase reveal the basis of flexible F1-Fo coupling. Science 364:eaaw9128. doi: 10.1126/science.aaw9128

Neumann, P., Dickmanns, A., and Ficner, R. (2018). Validating resolution revolution. Structure 26:1678. doi: 10.1016/j.str.2018.10.028

Niemelä, P. S., Miettinen, M. S., Monticelli, L., Hammaren, H., Bjelkmar, P., Murtola, T., et al. (2010). Membrane proteins diffuse as dynamic complexes with lipids. J. Am. Chem. Soc. 132, 7574-7575. doi: 10.1021/ja101481b

Nosé, S. (1984). A unified formulation of the constant temperature molecular dynamics methods. J. Chem. Phys. 81, 511-519. doi: 10.1063/1.447334

Oberfeld, B., Brunner, J., and Dimroth, P. (2006). Phospholipids occupy the internal lumen of the $\mathrm{c}$ ring of the ATP synthase of Escherichia coli. Biochemistry 45, 1841-1851. doi: 10.1021/bi052304+

Olsson, M. H. M., Søndergaard, C. R., Rostkowski, M., and Jensen, J. H. (2011). PROPKA3: consistent treatment of internal and surface residues in empirical pKa predictions. J. Chem. Theory Comput. 7, 525-537. doi: 10.1021/ct100578z

Ozaki, Y., Suzuki, T., Kuruma, Y., Ueda, T., and Yoshida, M. (2008). UncI protein can mediate ring-assembly of c-subunits of FoF1-ATP synthase in vitro. Biochem. Biophys. Res. Commun. 367, 663-666. doi: 10.1016/j.bbrc.2007.12.170

Parrinello, M., and Rahman, A. (1981). Polymorphic transitions in single crystals: a new molecular dynamics method. J. Appl. Phys. 52, 7182-7190. doi: $10.1063 / 1.328693$

Periole, X., Cavalli, M., Marrink, S.-J., and Ceruso, M. A. (2009). Combining an elastic network with a coarse-grained molecular force field: structure, dynamics, and intermolecular recognition. J. Chem. Theory Comput. 5, 2531-2543. doi: $10.1021 /$ ct9002114

Pettersen, E. F., Goddard, T. D., Huang, C. C., Couch, G. S., Greenblatt, D. M., Meng, E. C., et al. (2004). UCSF Chimera-a visualization system for exploratory research and analysis. J. Comput. Chem. 25, 1605-1612. doi: 10.1002/jcc.20084

Pogoryelov, D., Krah, A., Langer, J. D., Yildiz, Ö., Faraldo-Gómez, J. D., and Meier, T. (2010). Microscopic rotary mechanism of ion translocation in the F(o) complex of ATP synthases. Nat. Chem. Biol. 6, 891-899. doi: $10.1038 /$ nchembio. 457

Pogoryelov, D., Yu, J., Meier, T., Vonck, J., Dimroth, P., and Muller, D. J. (2005). The c15 ring of the Spirulina platensis F-ATP synthase: F1/F0 symmetry mismatch is not obligatory. EMBO Rep. 6, 1040-1044. doi: 10.1038/sj.embor.7400517

Qiu, W., Fu, Z., Xu, G. G., Grassucci, R. A., Zhang, Y., Frank, J., et al. (2018). Structure and activity of lipid bilayer within a membrane-protein transporter. PNAS 115, 12985-12990. doi: 10.1073/pnas.1812526115

Roh, S.-H., Stam, N. J., Hryc, C. F., Couoh-Cardel, S., Pintilie, G., Chiu, W., et al. (2018). The 3.5- $\AA$ CryoEM structure of nanodisc-reconstituted yeast vacuolar ATPase Vo proton channel. Mol. Cell 69, 993-1004.e3. doi: 10.1016/j.molcel.2018.02.006

Rühle, T., and Leister, D. (2015). Assembly of F1F0-ATP synthases. Biochim. Biophys. Acta 1847, 849-860. doi: 10.1016/j.bbabio.2015.02.005

Ryckaert, J.-P., Ciccotti, G., and Berendsen, H. J. C. (1977). Numerical integration of the cartesian equations of motion of a system with constraints: molecular dynamics of n-alkanes. J. Comput. Phys. 23, 327-341. doi: 10.1016/0021-9991(77)90098-5

Saroussi, S., Schushan, M., Ben-Tal, N., Junge, W., and Nelson, N. (2012). Structure and flexibility of the C-ring in the electromotor of rotary FoF1ATPase of pea chloroplasts. PLOS ONE 7:e43045. doi: 10.1371/journal.pone. 0043045

Schlegel, K., Leone, V., Faraldo-Gómez, J. D., and Müller, V. (2012). Promiscuous archaeal ATP synthase concurrently coupled to $\mathrm{Na}+$ and $\mathrm{H}+$ translocation. Proc. Natl. Acad. Sci. U.S.A. 109, 947-952. doi: 10.1073/pnas.11157 96109 
Schulz, S., Wilkes, M., Mills, D. J., Kühlbrandt, W., and Meier, T. (2017). Molecular architecture of the N-type ATPase rotor ring from Burkholderia pseudomallei. EMBO Rep. 18, 526-535. doi: 10.15252/embr.2016 43374

Sengupta, D., Rampioni, A., and Marrink, S.-J. (2009). Simulations of the c-subunit of ATP-synthase reveal helix rearrangements. Mol. Memb. Biol. 26, 422-434. doi: 10.3109/09687680903321073

Vollmar, M., Schlieper, D., Winn, M., Büchner, C., and Groth, G. (2009). Structure of the c14 rotor ring of the proton translocating chloroplast ATP synthase. J. Biol. Chem. 284, 18228-18235. doi: 10.1074/jbc.M109. 006916

Walker, J. E. (2013). The ATP synthase: the understood, the uncertain and the unknown. Biochem. Soc. Trans. 41, 1-16. doi: 10.1042/BST201 10773

Wassenaar, T. A., Ingólfsson, H. I., Böckmann, R. A., Tieleman, D. P., and Marrink, S. J. (2015). Computational lipidomics with insane: a versatile tool for generating custom membranes for molecular simulations. J. Chem. Theory Comput. 11, 2144-2155. doi: 10.1021/acs.jctc.5b00209

Wassenaar, T. A., Pluhackova, K., Böckmann, R. A., Marrink, S. J., and Tieleman, D. P. (2014). Going backward: a flexible geometric approach to reverse transformation from coarse grained to atomistic models. J. Chem. Theory Comput. 10, 676-690. doi: 10.1021/ct400617g

Webb, M. S., and Green, B. R. (1991). Biochemical and biophysical properties of thylakoid acyl lipids. Biochim. et Biophys. Acta Bioenerget. 1060, 133-158. doi: 10.1016/S0005-2728(09)91002-7
Wolf, M. G., Hoefling, M., Aponte-Santamaría, C., Grubmüller, H., and Groenhof, G. (2010). g_membed: Efficient insertion of a membrane protein into an equilibrated lipid bilayer with minimal perturbation. J. Comput. Chem. 31, 2169-2174. doi: 10.1002/jcc.21507

Zhou, W., Leone, V., Krah, A., and Faraldo-Gómez, J. D. (2017a). Predicted structures of the proton-bound membrane-embedded rotor rings of the Saccharomyces cerevisiae and Escherichia coli ATP synthases. J. Phys. Chem. B 121, 3297-3307. doi: 10.1021/acs.jpcb.6b 08051

Zhou, W., Marinelli, F., Nief, C., and Faraldo-Gómez, J. D. (2017b). Atomistic simulations indicate the c-subunit ring of the F1Fo ATP synthase is not the mitochondrial permeability transition pore. Elife 6:e23781. doi: $10.7554 /$ eLife. 23781

Conflict of Interest: The authors declare that the research was conducted in the absence of any commercial or financial relationships that could be construed as a potential conflict of interest.

Copyright (C) 2019 Novitskaia, Buslaev and Gushchin. This is an open-access article distributed under the terms of the Creative Commons Attribution License (CC BY). The use, distribution or reproduction in other forums is permitted, provided the original author(s) and the copyright owner(s) are credited and that the original publication in this journal is cited, in accordance with accepted academic practice. No use, distribution or reproduction is permitted which does not comply with these terms. 